\title{
Experiments on Microjets of Undercooled Liquid Hydrogen
}

\author{
José M. Fernández*, Matthias Kühnel ${ }^{\dagger}$, Guzmán Tejeda*, Anton Kalinin ${ }^{\dagger}$, \\ Robert E. Grisenti ${ }^{\dagger}$ and Salvador Montero* \\ * Laboratory of Molecular Fluid Dynamics, Instituto de Estructura de la Materia, CSIC, \\ Serrano 121, 28006 Madrid (Spain) \\ ${ }^{\dagger}$ Institut für Kernphysik, J. W. Goethe-Universität, Max-von-Laue str. 1, 60438 Frankfurt am Main (Germany)
}

\begin{abstract}
Novel experiments on liquid microjets (filaments) of hydrogen and deuterium, carried out at the Laboratory of Molecular Fluid Dynamics of the IEM, are reported. These filaments, less than 10 microns in diameter, are an ideal medium to produce highly undercooled liquid samples and to investigate the homogeneous solidification process, free from wall effects. The filaments exit from cryogenic capillary nozzles into a vacuum chamber, to cool down very fast by surface evaporation. Finite size radius leads to a temperature gradient across the filament, determined by thermal conductivity, and, possibly, to a velocity gradient as well. The filaments are monitored by laser shadowgraphy, and analyzed by means of high performance Raman spectroscopy. Real-time measurements in the rotational and vibrational spectral regions reveal the structure and temperature along the filaments, allowing to track the crystal growth process. The high spatial resolution of Raman spectroscopy allows observing in situ the structural changes of the liquid microjets, with a time resolution of $\sim 10$ ns. The filaments of pure para- $\mathrm{H}_{2}$ can be cooled down to $9 \mathrm{~K}(65 \%$ of its melting point at $13.8 \mathrm{~K})$, while staying liquid, before eventually solidifying into a metastable polymorph. Crystallization kinetics revealed a growth rate of $33 \mathrm{~cm} / \mathrm{s}$, much higher than expected for a thermally activated process. The time and spatial control attained in these experiments offers new opportunities for investigating the processes of nonequilibrium phase transformations in undercooled fluids, as well as the propagation of liquid jets into a rarefied gas media.
\end{abstract}

Keywords: Liquid microjets, Raman spectroscopy, undercooled hydrogen, deuterium, homogeneous solidification, crystalization kinetics PACS: $67.63 . \mathrm{Cd}, 33.20 . \mathrm{Fb}, 64.60 . \mathrm{My}, 64.70 . \mathrm{dg}, 47.60 . \mathrm{Kz}$

\section{INTRODUCTION AND MOTIVATION}

Hydrogen and its isotopes form the simplest closed-shell molecules, and $\mathrm{H}_{2}$ is the most prevalent molecule in the Universe. Due to nuclear spin statistics ${ }^{1} \mathrm{H}_{2}$ occurs as two species, with $I=0$ (para) and $I=1$ (ortho) respectively [1], which do not interconvert into each other but through magnetic interactions. At low temperatures, when all the population lie in its ground rotational state $J=0$, para $-\mathrm{H}_{2}$ is, like ${ }^{4} \mathrm{He}$, a spinless boson. Moreover, because of its low mass and weak van der Waals potential, hydrogen is expected to exhibit extensive quantum delocalization. Actually, quantum effects dominate the properties of its condensed phases [2,3], and para- $\mathrm{H}_{2}$ has been predicted to become superfluid at low enough temperatures [4].

The major obstacle to observing superfluidity of para $-\mathrm{H}_{2}$ is that the transition temperature, most recently estimated at about $2 \mathrm{~K} \mathrm{[5],} \mathrm{is} \mathrm{far} \mathrm{below} \mathrm{the} \mathrm{triple} \mathrm{point} \mathrm{at} 13.96 \mathrm{~K}$, as shown in Figure 1. Different strategies have been developed to overcome the solidification problem [6,7] without much success. Only indirect experimental evidence for superfluidity have been observed in para- $\mathrm{H}_{2}$ clusters, either inside an ultracold $(0.15 \mathrm{~K})$ helium droplet [8] or just nude [9].

Here, our approach for trying to avoid the crystal involves the fast undercooling of the liquid, taking advantage of the so called "nucleation island". According to classical nucleation theory, for a liquid to crystallize when cooled below its melting point, crystal nuclei need to be formed inside it, which eventually grow and extend to the whole volume. But this nucleation process takes some time to occur, so that, if the liquid is cooled fast enough, the system may remain liquid (undercooled) or become a glass.

\section{LIQUID FILAMENTS}

Liquid microjets (filaments) are produced by forcing a liquid to flow through a pinhole orifice, a few microns in diameter, into vacuum. Under laminar flow conditions the liquid propagates as a continuous cylindrical filament

28th International Symposium on Rarefied Gas Dynamics 2012

(C) 2012 American Institute of Physics 978-0-7354-1115-9/\$30.00 


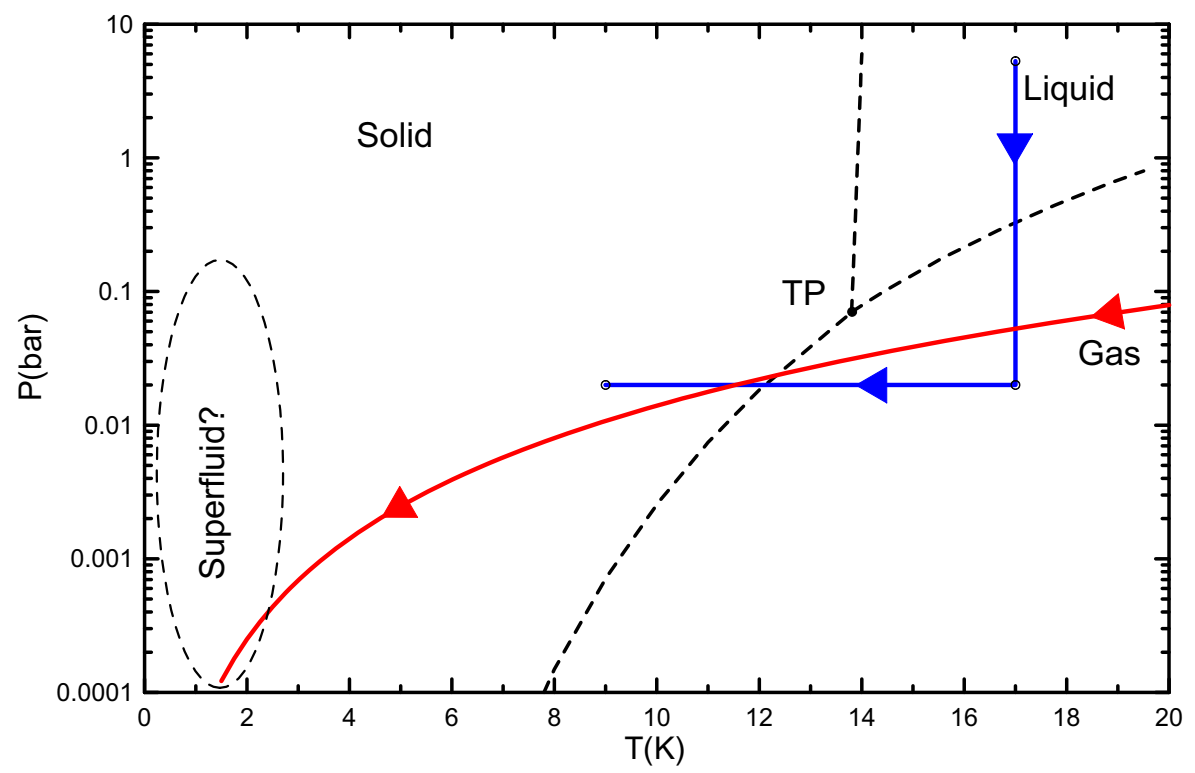

FIGURE 1. Phase diagram of $\mathrm{H}_{2}$ at low temperature, showing typical trajectories for an isentropic gas jet and a liquid jet in vacuum. TP stands for the triple pint at $T=13.96 \mathrm{~K}$ and $P=0.73$ bar.

until it eventualy breaks up into a monodirectional beam of droplets [10], as shown in Figure 2. The continuous filament provides a well-defined, container-free liquid sample of macroscopic dimensions, with a clean, continuously replenished surface, due to liquid evaporation. On that account, after being introduced by Faubel et al. [11], liquid filaments have found in recent years widespread applications [12, 13, 14, 15, 16, 17].

It is instructive to compare the properties of liquid jets in vacuum with those of the more familiar supersonic gas jets. The differences are due to the fact that gases are compressible fluids, while liquids are, in the present context, uncompressible in most practical terms.

- Density. For axisymmetric gas jets it falls with the square of distance to the nozzle, $n \sim 1 / z^{2}$, while for liquid jets is almost constant.

- Temperature. For gas jets it decreases with distance by the expansion work, which is done at expenses of its internal energy, reaching temperatures on the range of $\sim 10 \mathrm{~K}$ and below. Liquid jets in vacuum cool down by surface evaporation; evaporative cooling depends on the vapor pressure and latent heat of vaporization of the substance, and is intrinsically limited by the exponential decay in vapor pressure of the liquid with decreasing temperature. Thus, temperatures drops of $45 \mathrm{~K}$ can be attained for liquid $\mathrm{Ar}$, while only $10 \mathrm{~K}$ for liquid $\mathrm{O}_{2}$.

- Pressure. For gas jets is given by the equation of state $P=n R T$, and falls sharply with distance following $n$ and $T$. When a liquid jet of radius $r$ exits the nozzle, its pressure suddenly drops to the so-called Laplace pressure $P_{L}=2 \sigma / r$, determined by the surface tension $\sigma$ (responsible for the filament shape), and remains almost constant further downstream.

- Velocity. Supersonic gas jets accelerate up to a maximum velocity $v_{\max } \sim \sqrt{R T_{0} / m}$, determined by the stagnation temperature $T_{0}$ through the energy conservation principle. A liquid jet leaves the nozzle at a velocity determined by the stangation pressure $P_{0}$ and density, by means of the Bernoulli equation, $v=\sqrt{2 P_{0} / \rho}$, and remains almost constant.

- Metastable states. Both gas and liquid jets penetrate into new regions of the PT phase diagram under nonequilibrium conditions, as shown in Fig. 1, becoming thus metastable. This way, we can produce clusters and undercooled liquids, states non easily accesible by other means. 


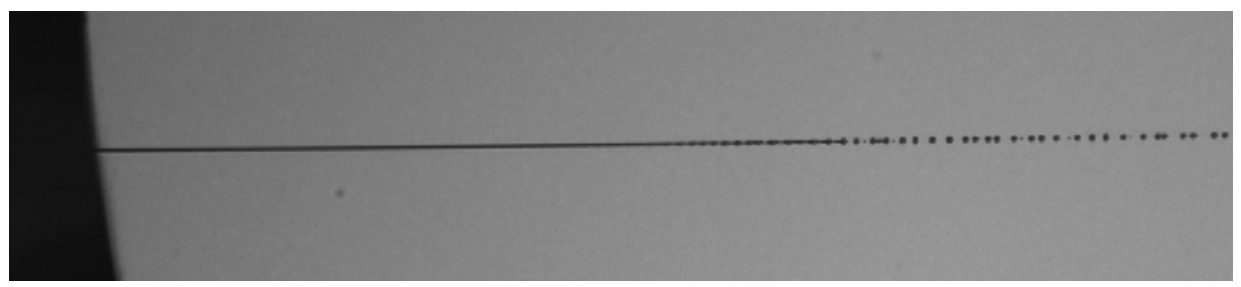

FIGURE 2. Micrography of a liquid $\mathrm{N}_{2}$ filament in vacuum, from a $4 \mu \mathrm{m}$ nozzle at 10 bar and $66 \mathrm{~K}$, showing the breakup into droplets.

\section{RAMAN SPECTROSCOPY OF $\mathrm{H}_{2}$ AND $\mathrm{D}_{2}$}

The Raman effect is the inelastic scattering of light (in the visible range) by matter. Raman scattering can be understood as a two-photon process, where an incident photon $v_{i}$ is absorbed by the sample and a second, different photon $v_{f}$ is emitted from the sample. In turn, the molecule undergoes a transition from an inital state $|i\rangle$ to a different one $|f\rangle$. The scattered radiation is emitted along all directions of space, though not with the same intensity nor polarization, and is unavoidly accompanyed by the much more intense Rayleigh (elastic) scattering.

In a typical experiment to observe the Raman spectrum, the sample is excited by means of an intense laser, and the scattered radiation is collected and analysed by means of some appropriate spectrometer. The Raman scattered photons bear information: i) on the energy level structure of the scatterer matter in its frequency, ii) on the density and population of the initial level of the transition, through its intensity, and iii) on the wavefunctions of the involved states, through its polarization.

Raman spectroscopy has some properties that make it very useful to probe molecular systems:

1. universality, in the sense that all molecular species give rise to Raman scattering,

2. high spatial resolution of a few microns, since the observable scattered radiation only comes from the sample volume where electric field irradiance in high enough,

3. intensity is strictly proportional to the population of the initial level, what allows to easily carry out quantitative diagnostics, and

4. broad spectral range, from $\sim 0$ to $\sim 6000 \mathrm{~cm}^{-1}$, what allows to record, with the same instrument, the spectra due to molecular rotational $\left(<350 \mathrm{~cm}^{-1}\right)$ or vibrational $\left(500-4500 \mathrm{~cm}^{-1}\right)$ transitions.

Nevertheless, the Raman effect is intrinsecally very weak, the scattering cross sections being of the order of $10^{-36} \mathrm{~m}^{2}$. Therefore, very sensitive instrumentation is needed to observe it with useful signal-to-noise ratios.

The Raman spectrum of $\mathrm{H}_{2}$ at low temperature is illustrated in Fig. 3. In addition to the usual vibrational $(\Delta v=1)$ transitions (right pannel), pure rotational $(\Delta J=2)$ transitions (left pannel) can also been observed. $\mathrm{H}_{2}$ is atypical in the sense that molecules can rotate almost freely in the condensed phases, and thus $J$ remains a good quantum number $[2,3]$. As can be seen in Fig. 3 right, the wavenumber of the vibrational line changes noticeably with the aggregation state; furthermore, the vibrational line position in the liquid is sensitive to the temperature [18], thus becoming a convenient thermal sensor. On the other hand, the rotational spectrum (Fig. 3 left) of the liquid is much broader than in the gas phase, due to the spread of the pair distribution function, but in the solid it is split into few components, depending on the symmetry (hexagonal or cubic) of the crystal field [3]. In summary, the Raman spectrum of $\mathrm{H}_{2}$ can provide direct, non-intrusive information on the aggregation state, temperature of the liquid, and structure of the solid phase.

\section{EXPERIMENTAL}

A scheme of the experimental set-up used in this work is shown in Fig. 4, a modification of that used in our previous work on gas jets $[19,20,21,22]$. High purity hydrogen $(99.9999 \%)$ and deuterium $(99.9 \%)$ were passed through catalytic ortho-para converters, mass-flow controllers for the preparation of gas mixtures, a system of pressure regulators, and a liquid nitrogen trap, before flowing into the nozzle inside the vacuum chamber. Nozzles were made of glass micropipettes, cut by natural break-up, and glued onto a copper piece in thermal contact with a liquid-helium 

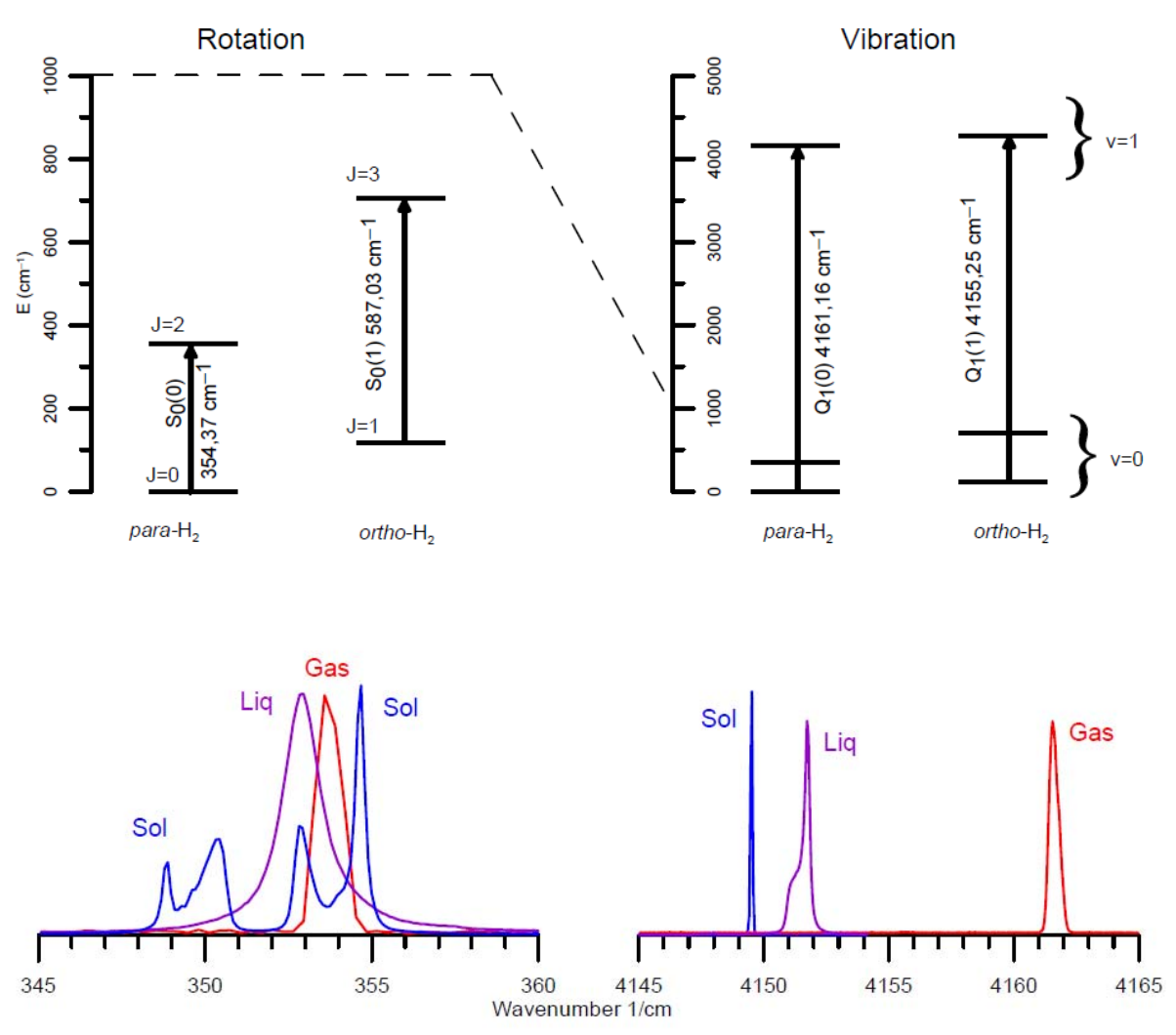

FIGURE 3. Raman spectroscopy of $\mathrm{H}_{2}$. Top: Rotational (left) and vibrational (right) energy levels. Bottom: Actual rotational (left) and vibrational (right) Raman spectra, showing their sensitivity to the aggregation state of the sample. Gas phase spectral lines in this figure are broadened by the instrument-limited resolution.

cryostat. The vacuum chamber, made in welded aluminum with dimensions $80 \times 40 \times 40 \mathrm{~cm}^{3}$, was evacuated by means of a 2000 liter/s turbomolecular pump backed by a $400 \mathrm{~m}^{3} /$ hour Roots blower and a $70 \mathrm{~m}^{3} /$ hour rotary pump, capable to keep the background pressure below $2 \times 10^{-3}$ mbar. Raman scattering from selected points along the liquid filament were excited at $\lambda=514.5 \mathrm{~nm}$ by a single-mode $\mathrm{Ar}^{+} \mathrm{cw}$-laser, Spectra Physics Beamlok, sharply focused by a $f=35 \mathrm{~mm}$ lens. Scattered radiation at 90 degrees was collected by a $f: 1.4$ photographic objective, and projected, with a total magnification $\times 9$, onto the entrance slit of a grating (2360 grooves $/ \mathrm{mm})$ spectrograph, equiped with a CCD detector refrigerated by liquid nitrogen. Nozzle holder, laser focusing optics, and Raman scattering collection optics inside the expansion chamber are mounted onto high-accuracy $( \pm 1 \mu \mathrm{m})$ xyz micropositioning actuators controlled from outside. A removable imaging system by laser shadowgraphy (LaVision), consisting in a pulsed frequencydoubled Nd:YAG laser plus a telescope and a fast CCD camera, was employed to monitor the filament appearance and stabilty. Working conditions were empirically established for each filament, but once successfully produced, they remained stable for hours.

An in-house design was used to cool the nozzle by means of the liquid-helium cryostat. The nozzle must be in thermal contact with the cold tip of the cryostat, while at the same time being movable with sub one-micron accuracy. All this was accomplished by using an U-shaped copper strap to link the nozzle with the cryostat, combined with a pair of glass blocks to thermally isolate the nozzle holder from its micropositioning actuators. With this design, the nozzle could be cooled down to $10 \mathrm{~K}$.

In order to work with the pure low-energy $\left(I=\right.$ even) nuclear spin species of $\mathrm{H}_{2}$ and $\mathrm{D}_{2}$, two home-built ortho-para converters were used in continuous flow conditions. To accomplish the ortho-para conversion, the hydrogen gases are cooled down to the liquid phases in the presence of a magnetic catalyst, to drag all the population towards the $J=0$ lowest energy state. Closed-cycle helium cryocoolers were used for the cooling, and iron(III) hydrated oxide (Aldrich 


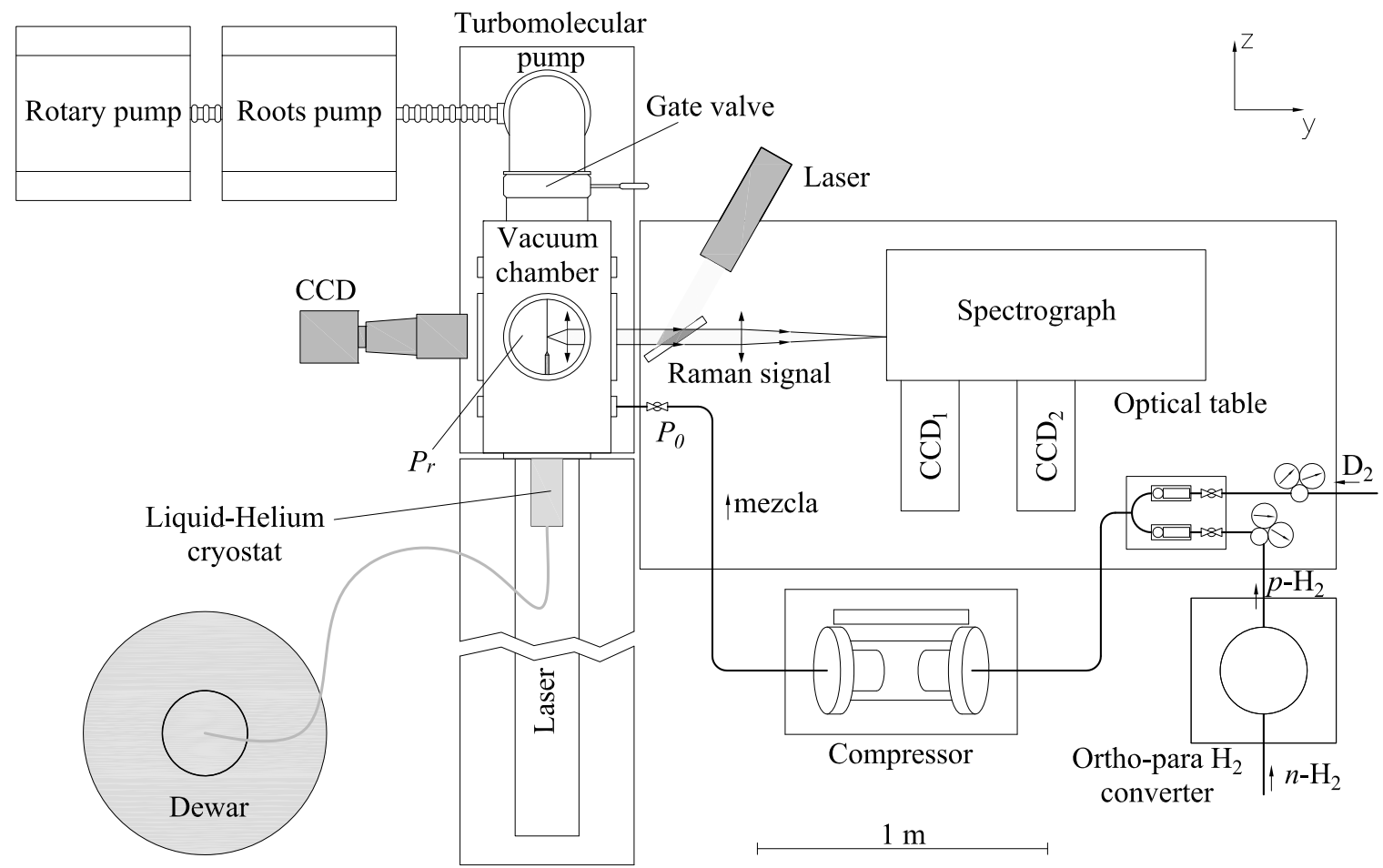

FIGURE 4. Schematic view of the experimental setup from the top.

No. 371254) as catalyst. Temperatures of $17 \mathrm{~K}$ and $21 \mathrm{~K}$ were used for $\mathrm{H}_{2}$ and $\mathrm{D}_{2}$, respectively, yielding conversions of $99.99 \%$ and $98.5 \%$, under flows up to $1000 \mathrm{sccm}$. Once converted, the $I=$ even species thus obtained can be stored and used at room temperature without significant back-conversion in the absence of magnetic substances.

\section{RESULTS}

A series of experiments on filaments of liquid para $-\mathrm{H}_{2}$, ortho- $\mathrm{D}_{2}$, and mixtures of para- $\mathrm{H}_{2}$ and ortho- $\mathrm{D}_{2}$ with different mixing ratios, as summarized in Table 1, were carried out. Series of Raman spectra along the filament axis were recorded in the following spectral regions: rotation of ortho- $\mathrm{D}_{2}$ at $180 \mathrm{~cm}^{-1}$, rotation of para- $\mathrm{H}_{2}$ at $354 \mathrm{~cm}^{-1}$, vibration of ortho- $\mathrm{D}_{2}$ at $2890 \mathrm{~cm}^{-1}$, and vibration of para $-\mathrm{H}_{2}$ at $4150 \mathrm{~cm}^{-1}$. The results for the pure substances are discussed next.

\section{para $-\mathbf{H}_{2}$}

A series of rotational and vibrational Raman spectra of a liquid microjet of para- $\mathrm{H}_{2}$ recorded between $z=0.03 \mathrm{~mm}$ and $z=2.03 \mathrm{~mm}$ is displayed in Figure 5 [23]. At the closest spot to the nozzle orifice, the rotational spectrum shows the broad line of the liquid, while the vibrational spectrum consists of a strongly asymmetric band with its peak centered at $4151.7 \mathrm{~cm}^{-1}$, which corresponds to bulk liquid para $-\mathrm{H}_{2}$ at $T=16.8 \mathrm{~K}$. As long as we move farther away from the nozzle, the vibrational line of the liquid shifts to lower wavenumbers, indicating a cooling of the filament [18]. At $z>0.9 \mathrm{~mm}$ additional, rapidly growing peaks appear in the rotational and vibrational spectra, corresponding 


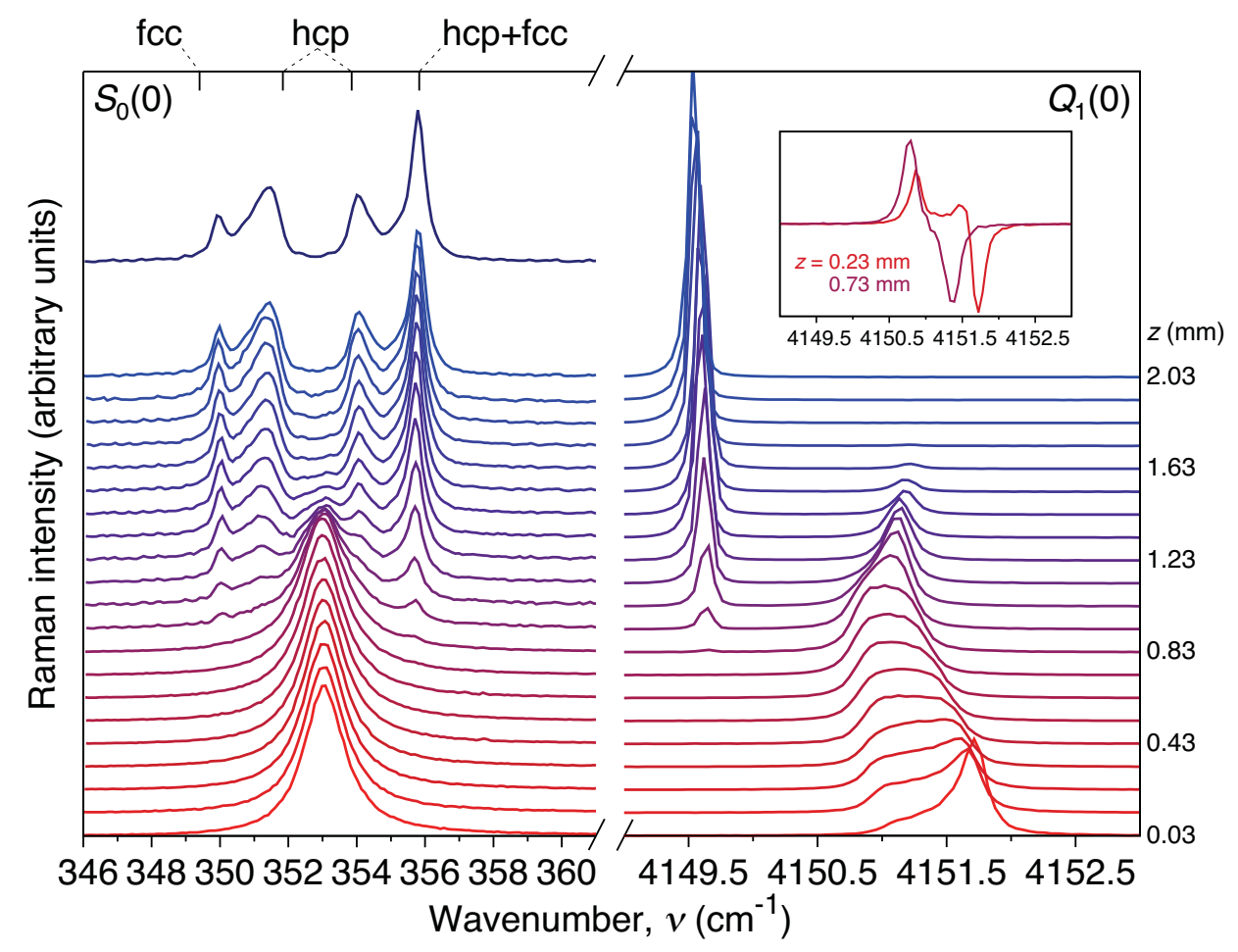

FIGURE 5. Rotational (left) and vibrational (right) Raman spectra of a filament of para- $\mathrm{H}_{2}$ along its axis from a $5 \mu \mathrm{m}$ nozzle at 14 bar and $16 \mathrm{~K}$ [23]. The ticks on the top axis of left pannel mark the wavenumbers for the hcp and fcc structures in bulk para- $\mathrm{H}_{2}$ [23].

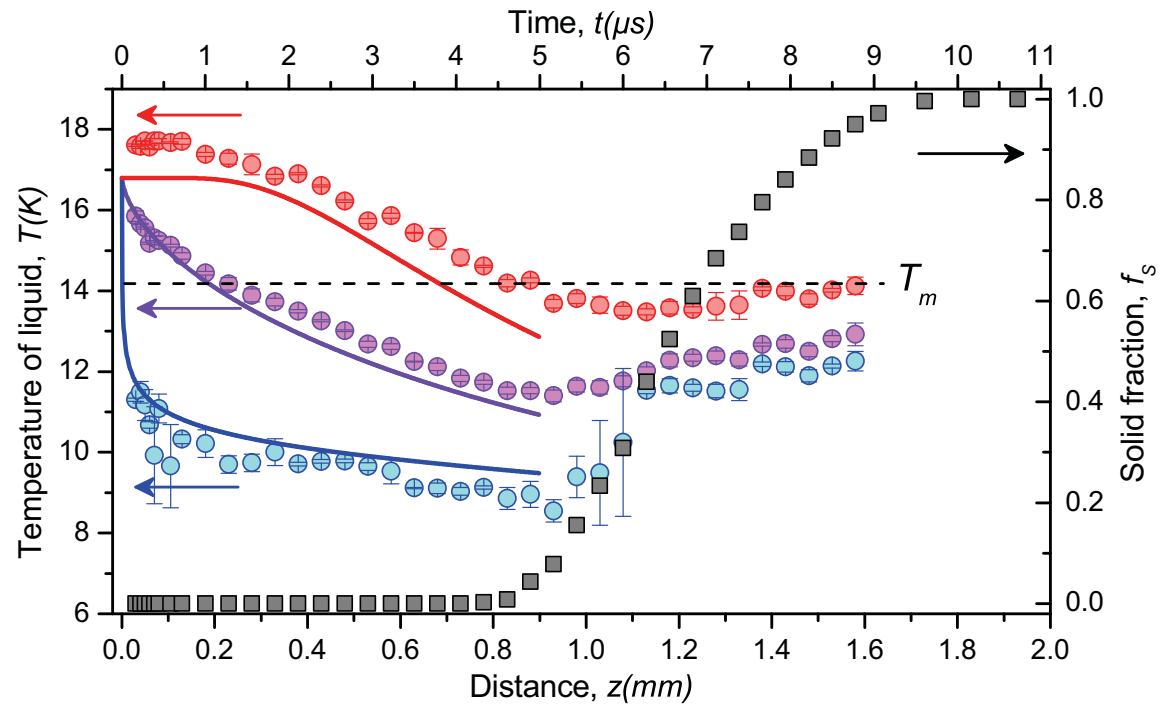

FIGURE 6. Solid fraction (right axis) and temperature of the liquid fraction (left axis) of the filament of Fig. 5 along distance from the nozzle (bottom axis). Circles are experimental temperatures extracted from the correlation of Ref. [18], while solid lines are calculated from a multilayer model. $T_{m}$ marks the normal melting point of $p a r a-\mathrm{H}_{2}$ at $13.8 \mathrm{~K}$. Distances have been converted into times (top axis) through the jet velocity $v=180 \mathrm{~m} / \mathrm{s}$. 
TABLE 1. Liquid microjets investigated in the present work.

\begin{tabular}{ccrr}
\hline nozzle $(\mu \mathbf{m})$ & composition & $P_{0}$ (bar) & $T_{0}(\mathbf{K})$ \\
\hline 5 & para $-\mathrm{H}_{2}$ & 13.8 & 15.8 \\
5 & $3 \% o \mathrm{D}_{2}+97 \% p \mathrm{H}_{2}$ & 8.0 & 16.0 \\
5 & $17 \% o \mathrm{D}_{2}+83 \% p \mathrm{H}_{2}$ & 11.0 & 17.0 \\
5 & $52 \% o \mathrm{D}_{2}+48 \% p \mathrm{H}_{2}$ & 13.5 & 17.0 \\
5 & $84 \% o \mathrm{D}_{2}+16 \% p \mathrm{H}_{2}$ & 13.5 & 19.0 \\
5 & ortho $-\mathrm{D}_{2}$ & 12.0 & 19.6 \\
\hline 2 & para $-\mathrm{H}_{2}$ & 22.6 & 16.5 \\
2 & $15 \% o \mathrm{D}_{2}+85 \% p \mathrm{H}_{2}$ & 14.0 & 16.0 \\
2 & $50 \% o \mathrm{D}_{2}+50 \% p \mathrm{H}_{2}$ & 24.2 & 18.9 \\
2 & ortho $-\mathrm{D}_{2}$ & 24.6 & 20.0 \\
\hline \multicolumn{4}{c}{} \\
\hline
\end{tabular}

to solid para $-\mathrm{H}_{2}[18,24]$. This is accompanied by a temperature increase of the liquid and thus results from the heat released during the solidification process.

The average filament temperature as a function of distance to the nozzle $z$ (middle circles), is plot in Figure 6, illustrating the rapid cooling of the liquid filament in vacuum. The broad shape of the vibrational band of the liquid in Fig. 5-right, especially seen during early propagation times, is the signature of a temperature gradient across the liquid stream. This is illustrated more explicitly in Fig. 6 by the large temperature gap for $z<1 \mathrm{~mm}$ between the hottest (upper circles) and coldest (lower circles) regions of the filament, which correspond to the two extreme inflection-point wave numbers in the vibrational band of the liquid (see the inset in Fig. 5-right).

The radial temperature gradient results from the finite thermal conductivity of liquid hydrogen. To show this, we simulate the jet cooling by dividing the filament into 100 equally mass-weighted cylindrical layers. We allow for evaporative cooling [25] of the outermost layer, whereas the heat transfer between any two neighboring layers is determined by thermal conductivity. The computed filament temperature averaged over the entire volume, on one hand, and the temperatures of the inner core and surface layer, on the other hand, are shown in Fig. 6 as middle, upper and lower solid lines, respectively. The agreement with the experimental data also confirms the deep quenching to about $9 \mathrm{~K}\left(\sim 0.65 T_{m}\right)$ of a fraction of the expanding liquid filament. The steep increase of the filament surface temperature for $z>0.9 \mathrm{~mm}$ suggests that the crystallization front initiates at the cold surface and proceeds inwards. This is further confirmed by additional vibrational Raman spectra recorded transversely to the filament axis, which show that the solid fraction at the filament edge is larger than at the center.

The solid fraction $f_{S}$ at the probed spot can be estimated from the ratio of the intensity of the peak at $4149 \mathrm{~cm}^{-1}$ with respect to the total intensity of the vibrational spectrum. The time evolution of this solid fraction, shown in Fig. 6 , allows us to estimate the rate of linear crystal growth $u$, which is the fundamental physical quantity governing the kinetics of crystallization [26]. A lower limit for the growth rate is readily obtained by dividing the filament radius ( $r=2.5 \mu \mathrm{m}$ ) by the total freezing time $(8 \mathrm{~s})$, yielding $u=33 \mathrm{~cm} / \mathrm{s}$ [23]. This crystal growth rate is two orders of magnitude larger than that calculated for a thermally activated process, but it is consistent with a collision-limited model, with a characteristic rate determined by the average thermal velocity

$$
u_{0}=\sqrt{3 k T / m}
$$

Additional insights into the crystallization process can be derived from the analysis of the rotational spectra in Fig. 5left. The rotational Raman spectrum of bulk solid para $-\mathrm{H}_{2}$ consists of either three lines, corresponding to the hexagonal closed-packed (hcp) crystal structure, or two lines, which correspond to the face-centered cubic (fcc) structure [3]. Solid para- $\mathrm{H}_{2}$, when grown slowly from the melt, is always found to be hcp [2], which is the energetically favored crystal structure. However, our spectra clearly evidence four peaks, which agree with both the hcp and fcc structures, indicating that the rapidly cooled liquid crystallizes into a metastable polymorph, a random mixture of hcp and fcc domains.

The 2-micron filaments confirm these results, though with a larger filament velocity because of the higher pressures employed (see Table 1). The same spectral patterns of Figure 5 are observed, but the cooling and crystalization kinetics are much faster. This is due to the smaller diameter, what causes the temperature gradients to be smaller as well, and the crystal growth front has to travel a shorter distance from the surface layer to the inner core. 

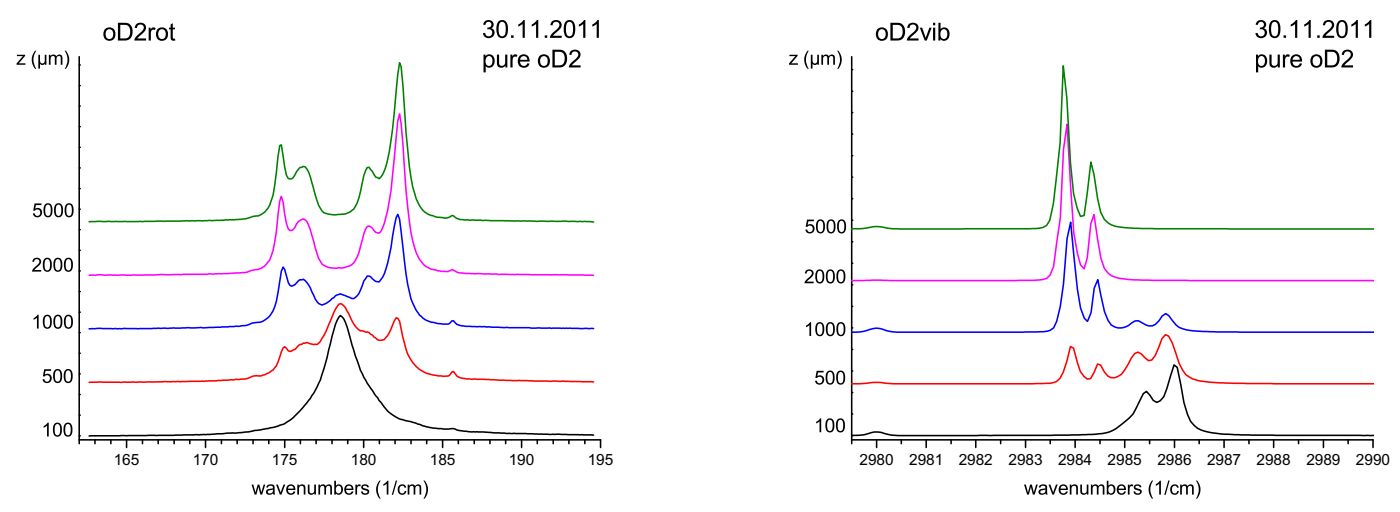

FIGURE 7. Rotational (left) and vibrational (right) Raman spectra of a liquid ortho- $\mathrm{D}_{2}$ filament at increasing distances from a $5 \mu \mathrm{m}$ nozzle at 12 bar and $19.6 \mathrm{~K}$.

\section{ortho- $\mathbf{D}_{2}$}

The Raman spectra of the filaments of pure ortho- $\mathrm{D}_{2}$ in Figure 7 show a trend much similar to that of the pure para- $\mathrm{H}_{2}$, being liquid close to the nozzle until they eventually crystallize, without breaking up into droplets. Thus, the rotational spectrum in Figure 7 left show a transition from the broad line of the liquid to the characteristic four peak pattern of the solid, caused by the overlapping of the hcp and fcc crystal field splittings. However, two differences deserve mentioning. First, the crystallization kinetics is slower in ortho- $\mathrm{D}_{2}$ than in para- $\mathrm{H}_{2}$, the crystallization time being $\sim \sqrt{2}$ larger, what is consistent with a collision-limited process with a characteristic rate determined by the average thermal velocity of Eq. (1). And second, because of the impossibility to prepare $100 \%$ pure ortho- $\mathrm{D}_{2}$ the vibrational Raman spectra of both liquid and solid consist of doublets, corresponding to $J=0$ and residual $(\sim 1.5 \%)$ $J=1$ lines, as opposed to the single line observed in pure para $-\mathrm{H}_{2}$. Moreover, due to the coupling of the vibrational motions of neighboring molecules, a remarkable enhancement of the Raman intensity of the $J=1$ minor component occurs, which is stronger in the solid [27]: note the peak of the $(\sim 1.5 \%) J=1$ species at $2983.8 \mathrm{~cm}^{-1}$ is more intense than that of the most abundant $J=0$ one at $2984.4 \mathrm{~cm}^{-1}$. The complete analysis of the crystalization kinetics of all the mixtures listed in Table 1, and of the changes in the spectra as a function of the mixture composition are currently under way.

\section{SUMMARY AND CONCLUSIONS}

Filaments (microjets) propagating into vacuum have been shown to be an ideal medium to produce samples of deeply undercooled liquids. Combined with high-performance Raman spectroscopy, these microjets provide a powerful technique to study very fast processes, like the general phenomenon of homogeneous crystalization, free from walleffect perturbances. The technique has been applied here to the particular case of hydrogen and deuterium, the simplest molecular substances, where Raman spectroscopy can provide non-intrusive information on aggregation state, structure, and temperature of the liquid, with a time resolution of $\sim 10 \mathrm{~ns}$. In the experiments reported in this work, para $-\mathrm{H}_{2}$ has been undercooled to $\sim 9 \mathrm{~K}$ before crystalizing into a polymorph, being able to measure a crystal growth speed of $30 \mathrm{~cm} / \mathrm{s}$, the faster ever measured. Some of the structures observed in this work are only accesible under these fast growing conditions.

Despite the deep undercooling reached in this work for para $-\mathrm{H}_{2}$, we are still far away from the predicted temperature for the superfluid transition. Future extensions of the present experiments will include the use of buffer gas cooling. This may be accomplishced either by propagating the filament through a static gas in a cold cell, or inside a supersonic gas jet using a concentric nozzle assembly, like those used to focus capillary jets [28].

The propagation of these filaments in vacuum raises some problems of interest to the rarefied gas dynamics. The flow field of the evaporated gas around the filament, with $\mathrm{Kn}>0.1$, lies within the transition or molecular flow regimes. To this regard, as pointed out previously, the influence of the surrounding rarefied gas on the filament cooling is of 
first-rank practical interest, and whether that can be used to enhace or impede the filament cooling. Finally, it would be highly desirable to know the influence of the nozzle shape and its surface finishing on the stability of the filaments, what might open new possibilities to control their properties.

\section{ACKNOWLEDGMENTS}

This work has been supported by the the Spanish Ministerio de Ciencia e Innovacion, through grants FIS2007-61430, FIS2010-22064-C02-01, and HD2008-0068, by the Helmholtz Gemeinschaft, through grant VH-NG-331, and by the German academic exchange service (DAAD) under reference Nr. 50025171.

\section{REFERENCES}

1. The names ortho and para obey a historical convention, ortho being used for the most abundant species and para for the least abundant. In the case of $\mathrm{H}_{2}$ and $\mathrm{D}_{2}$ the lowest energy $(I=$ even $)$ species are para- $\mathrm{H}_{2}$ and ortho- $\mathrm{D}_{2}$, respectively.

2. I. F. Silvera, Rev. Mod. Phys. 52, 393-452 (1980).

3. J. van Kranendonk, Solid Hydrogen (Plenum Press, New York, 1983).

4. V. L. Ginzburg and A. A. Sobyanin, JETP Lett. USSR 15, 242 (1972).

5. V. S. Vorob'ev and S. P. Malyshenko, J. Phys. Condens. Matter 12, 5071-5085 (2000).

6. H. J. Maris, G.M. Seidel, and F. I. B. Williams, Phys. Rev. B 36, 6799-6810 (1987).

7. M. Schindler, A. Dertinger, Y. Kondo, and F. Pobell, Phys. Rev. B 53, 11451-11461 (1996).

8. S. Grebenev, B. Sartakov, J. P. Toennies, and A. F. Vilesov, Science 289, 1532-1535 (2000).

9. P. C. Raston, W. Jäger, H. Li, R. J. LeRoy, and P. N. LeRoy, Phys. Rev. Lett. 108, 253402 (2012).

10. A. Frohn and N. Roth, Dynamics of Droplets (Springer, Berlin, 2000).

11. M. Faubel, S. Schlemmer, and J. P. Toennies, Z. Phys. D 10, 269-277 (1988)

12. M. Berglund, L. Rymell, H. M. Hertz, and T. Wilhein, Rev. Sci. Instrum. 69, 2361-2364 (1998).

13. M. Faubel, in Photoionization and Photodetachement, edited by C. Y. Ng (World Scientific, Singapore, 2000).

14. R. E. Grisenti and J. P. Toennies, Phys. Rev. Lett. 90, 234501 (2003).

15. J. D. Smith, C. D. Cappa, K. R. Wilson, B. M. Messer, R. C. Cohen, and R. J. Saykally, Science 306, 851-853 (2004).

16. U. Weierstall, J. C. Spence, and R. B. Doak, Rev. Sci. Instrum. 83, 035108 (2012).

17. R. A. Costa-Fraga, A. Kalinin, M. Kühnel, D. C. Hochhaus, A. Schottelius, J. Polz, M. C. Kaluza, P. Neumayer, and R. E. Grisenti, Rev. Sci. Instrum. 83, 025102 (2012).

18. R. Sliter and A. F. Vilesov, J. Chem. Phys. 131, 074502 (2009)

19. S. Montero, B. Maté, G. Tejeda, J. M. Fernández, and A. Ramos, in Atomic and Molecular Beams. The State of the Art 2000, edited by R. Campargue, Springer, Berlin, 2001, pp. 295-306.

20. G. Tejeda, J. M. Fernández, S. Montero, D. Blume, and J. P. Toennies, Phys. Rev. Lett. 92, 223401 (2004).

21. S. Montero, J. H. Morilla, G. Tejeda, and J. M. Fernández, Eur. Phys. J. D 52, 31-34 (2009).

22. J. H. Morilla, J. M. Fernández, G. Tejeda, and S. Montero, Phys. Chem. Chem. Phys. 12, 12060-12064 (2010)

23. M. Kühnel, J. M. Fernández, G. Tejeda, A. Kalinin, S. Montero, and R. E. Grisenti, Phys. Rev. Lett. 106, 245301 (2011).

24. S. S. Bhatnagar, E. J. Allin, and H. L. Welsh, Can. J. Phys. 40, 9-23 (1962).

25. R. E. Grisenti, R. A. Costa-Fraga, N. Petridis, R. Dörner, and J. Deppe, Europhys. Lett. 73, 540-546 (2006).

26. K. A. Jackson, Kinetic Processes (Wiley, Weinheim, 2004).

27. H. M. James and J. van Kranendonk, Phys. Rev. 164, 1159-1168 (1967).

28. A. M. Gañán-Calvo, R. González-Prieto, P. Riesco-Chueca, M. A. Herrada, and M. Flores-Mosquera, Nature Phys. 3, 737-742 (2007). 